\title{
NUCLEAR vs NUCLEON STRUCTURE EFFECTS ON NUCLEAR TRANSPARENCY
}

\author{
OMAR BENHAR ${ }^{a}$ \\ Jefferson Lab Theory Group, Newport News, VA 23606 \\ and \\ Physics Department, Old Dominion University, Norfolk, VA 23529
}

\begin{abstract}
Nuclear structure effects account for the observed enhancement of the nuclear transparency to moderate energy protons, with respect to the predictions of Glauber theory. This enhancement appears to be comparable to the one associated with the onset of color transparency in the $Q^{2}$ range spanned by the available $\left(e, e^{\prime} p\right)$ data $\left(Q^{2}<7(\mathrm{GeV} / \mathrm{c})^{2}\right)$. It is argued that in this kinematical regime a stronger colour transparency signal can be observed in the low energy loss tail of the inclusive electron-nucleus cross section, corresponding to large values of the Bjorken scaling variable $x(x>2)$.
\end{abstract}

\section{Introduction}

According to quantum-chromo-dynamics (QCD), pointlike colourless systems, such as those produced in quasielastic scattering at high $Q^{2}$, exhibit vanishingly small hadronic cross sections. Therefore, they are expected to travel through nuclear matter experiencing very little attenuation. Over the last five years, the possible manifestation of this effect, generally referred to as colour transparency (CT), in the process $e+A \rightarrow e^{\prime}+p+X$ has been the focus of a significant amount of experimental and theoretical activity. The basic observable carrying the signature of $\mathrm{CT}$ in coincidence $\left(e, e^{\prime} p\right)$ reactions off nuclear targets is the transparency ratio:

$$
T_{A}\left(Q^{2}\right)=\frac{\sigma_{e x p t}\left(Q^{2}\right)}{\sigma_{P W I A}\left(Q^{2}\right)},
$$

where $Q^{2}$ denotes the virtuality of the exchanged photon, $\sigma_{\text {expt }}$ is the measured cross section and $\sigma_{P W I A}$ is the cross section in absence of final state interactions (FSI) leading to the absorption of the knocked-out proton (notice that the possibility of obtaining $T_{A}$ from the above definition in a model independent way is somewhat questionable, since the full knowledge of the nuclear spectral function is required to compute the denominator). Within the colour transparency picture, at large enough $Q^{2} T_{A}\left(Q^{2}\right)$ is expected to become an increasing function of $Q^{2}$, and eventually reach the asymptotic value $T_{A}=1$, corresponding to zero absorption.

\footnotetext{
${ }^{a}$ On leave from INFN, Sezione Sanità. I-001161 Rome, Italy
} 
Unfortunately, the available data ${ }^{1,2}$ only cover the region of moderate $Q^{2}\left(Q^{2}<7(\mathrm{GeV} / \mathrm{c})^{2}\right)$, where $\mathrm{CT}$ is expected to produce a rather small enhancement of $T_{A}$, of the order of 10-20\%. Therefore, in order to meaningfully interpret the data, the nuclear absorption of the outgoing proton in absence of colour transparency must be under control to high accuracy, and calculations of the nuclear transparency carried out within standard nuclear many-body theory have to be regarded as a prerequisite for the experimental analysis.

A rough estimate of the mean-free-path of a proton of energy $E$ travelling through nuclear matter can be given in terms of the matter density $\rho$ and the proton-nucleon $(p \mathrm{~N})$ cross section $\sigma$ :

$$
\lambda=\frac{1}{\sigma \rho} .
$$

At $E=200 \mathrm{MeV}$ the above equation yields $\lambda \sim 2.5 \mathrm{fm}$, to be compared with the result based on optical model fits:

$$
\lambda=\frac{[(E-V) / 2 m]^{1 / 2}}{W},
$$

where $U$ and $W$ denote the real and imaginary part of the nuclear matter optical potential, respectively, and $m$ is the nucleon mass. At $E \sim 100-200$ $\mathrm{MeV}$ eq.(3) yields $\lambda \sim 5-7 \mathrm{fm}$, showing that the interactions responsible for proton absorption cannot be described in terms of free-space cross section and average density. In order to get accurate estimates of the mean-free-path, more complex nuclear structure effects, leading to local density fluctuations and to modifications of the nucleon-nucleon (NN) scattering cross section, have to be carefully taken into account.

\section{Many-body calculation of the nuclear transparency}

Theoretical calculations of $T_{A}\left(Q^{2}\right)$ can be carried out within the framework of nuclear many-body theory using a generalization of Glauber theory of proton scattering ${ }^{3,4,5}$. The basic assumptions involved in this approach are: i) the eikonal approximation, implying that the knocked-out nucleon moves along a straight trajectory with constant velocity $\mathbf{v}$, and ii) the frozen approximation, which amounts to treating the spectator nucleons as a collection of fixed scattering centers. The resulting expression for a proton of momentum $P$ reads

$$
T_{A}=\int d R\left|\Psi_{A}(R)\right|^{2}\left|\Omega_{P}(R)\right|^{2}
$$


with

$$
\Omega_{P}(R)=\frac{1}{A} \sum_{i=1}^{A} \mathrm{e}^{i \sum_{j \neq i=1}^{A} \int_{0}^{\infty} d t^{\prime} w_{P}\left(\left|\mathbf{r}_{i}-\mathbf{r}_{j}+\mathbf{v} t^{\prime}\right|\right)},
$$

where $R \equiv\left\{\mathbf{r}_{1}, \mathbf{r}_{2}, \ldots, \mathbf{r}_{A}\right\}$ specifies the target configuration, $\Psi_{A}(R)$ is the target ground state wave function, $\mathbf{v}=\mathbf{P} / m$ and the two body interaction $w_{P}(r)$ is defined in terms of the NN scattering amplitude $f_{P}(k)$ as

$$
w_{P}(r)=\frac{2 \pi}{m} \int \frac{d^{3} k}{(2 \pi)^{3}} \mathrm{e}^{\imath \mathbf{k} \cdot \mathbf{r}} f_{P}(k) .
$$

The free space $f_{P}(k)$ can be obtained from a three-parameter fit to the data in the form:

$$
f_{P}(k)=\frac{P}{4 \pi}\left(i+\alpha_{P}\right) \sigma \mathrm{e}^{-\beta_{P}^{2} k^{2}} .
$$

In the nuclear medium, the measured scattering cross section $\sigma$ has to be replaced with an effective cross section $\sigma_{\text {eff }}<\sigma$, to take into account Pauli blocking, constraining the angular distribution of the scattered nucleons, and the modification of the density of final states due to the fact that bound nucleons have an effective mass $m_{e f f}<m$. In fig. 1 the free space NN cross section (solid line) is compared to $\sigma_{\text {eff }}$ calculated in infinite nuclear matter at equilibrium density $\left(\rho=.16 \mathrm{fm}^{-3}\right.$ ) (dashed line), as a function of the energy of the beam particle in the laboratoty frame ${ }^{6}$. It clearly appears that the presence of the nuclear medium strongly affects NN scattering, leading to a sizeable reduction of the total cross section that persists even at large energy.

Expanding the exponential factor in eq.(5), eq.(4) can be rewritten as

$$
T_{A}=\sum_{n=0}^{A-1} T_{A}^{(n)}
$$

where $T_{A}^{(n)}$ represents the contribution to $T_{A}$ from processes involving $n$ rescatterings (obviously $T_{A}^{(0)}=1$ ). The rescattering probability is strongly affected by the density fluctuations induced by the repulsive core of the NN force. For intance, the (negative) single rescattering term, sensitive to correlations between the projectile particle and the spectators, turns out to be significantly suppressed by the "hole" at small $\left|\mathbf{r}_{i}-\mathbf{r}_{j}\right|$ in the two-body distribution function

$$
p^{(2)}\left(\mathbf{r}_{i}, \mathbf{r}_{j}\right)=\rho_{A}\left(\mathbf{r}_{i}\right) \rho_{A}\left(\mathbf{r}_{j}\right) g\left(\mathbf{r}_{i}, \mathbf{r}_{j}\right),
$$

yielding the joint probability of finding two nucleons at positions $\mathbf{r}_{i}$ and $\mathbf{r}_{j}$ in the target ground state. In absence of correlations, the $n$-body distribution function $p^{(n)}\left(\mathbf{r}_{i_{1}}, \ldots, \mathbf{r}_{i_{n}}\right)$ reduces to the products of $n$ one-body densities $\rho_{A}\left(\mathbf{r}_{i}\right)$, and the standard result of Glauber theory is recovered. 
The A-dependence of the single- and double-rescattering contributions to $T_{A}$ is illustrated in fig. 2 for the case of a $600 \mathrm{MeV}$ nucleon. The calculations have been carried out using the empirical nuclear densities extracted from elastic electron-nucleus scattering data ${ }^{7}$ and the infinite nuclear matter $g(r)$ to generate the two- and three-body distribution functions within the local density approximation. The enhancement of $T_{A}$ with respect to the prediction of Glauber theory, produced by NN correlations, is $\sim 20 \%$ in ${ }^{12} \mathrm{C}$ and $\sim 15 \%$ in ${ }^{208} \mathrm{~Pb}$.

For the case of ${ }^{16} \mathrm{O}$, the calculation of $T_{A}$ has been recently carried out from eq.(4), i.e. including all the rescattering terms, using the many-body wave function resulting from the variational calculation of Pieper $e t a l^{8}$. The result of this calulation ${ }^{9}$ is shown in fig. 3 as a function of the momentum of the knocked-out nucleon.

\section{Data}

Fig. 4 shows the comparison between the effective cross section $\widetilde{\sigma}$ extracted from the analysis of the A-dependence of the SLAC NE18 data ${ }^{1}$ in terms of semiclassical attenuation, i.e. assuming

$$
T_{A}=\frac{1}{A} \int d^{3} r \rho_{A}(\mathbf{b}, z) \mathrm{e}^{-\int_{z}^{\infty} d z^{\prime} \rho_{A}\left(\mathbf{b}, z^{\prime}\right)},
$$

whith $\mathbf{r}=\mathbf{b}+(\mathbf{v} / \mathrm{v}) z$, and the measured free-space NN total cross section. As expected on the basis of the analysis of nuclear structure effects carried out in the previous section, the in medium cross section is appreciably smaller than the free cross section. Whether or not part of the difference between $\sigma$ and $\widetilde{\sigma}$ has to be ascribed to the onset of colour transparency can only be assessed studying the $Q^{2}$ dependence of the data. Unfortunately, the data of fig. 4 are rather inconclusive in this respect, since the difference $\sigma-\widetilde{\sigma}$ is almost constant at $Q^{2}=3$ and $5(\mathrm{GeV} / \mathrm{c})^{2}$, while showing only a modest increse at the highest $Q^{2}=6.8(\mathrm{GeV} / \mathrm{c})^{2}$.

Fig. 5 shows a comparison between the result of a many-body calculation of $T_{A}$ including the two- and three-body rescattering terms, and the available data, including the low-energy data point from the MIT-Bates exeriment of Garino $e t a l^{10}$. It appears that, although the possibility of a small CT effect cannot be ruled out, the conventional nuclear physics picture provides a fairly good description of the measured transparencies. 


\section{Conclusions and prospects}

The results of realistic calculations carried out within microscopic many-body theory show that in the region of moderate $Q^{2}$, covered by the available $\left(e, e^{\prime} p\right)$ data, the measured nuclear transparency can be interpreted in terms of nuclear structure effects, leading to a suppression of the NN cross section and to strong local fulctuations in the density of the spectator nucleons. A clearcut evidence of CT could only be obtained extending the measurement of $T_{A}$ to larger values of $Q^{2}$.

As a final remark, it is worthwhile mentioning that observables other than nuclear transparency are expected to be very sensitive to CT even at moderate $Q^{2}$. The low energy loss tail of the inclusive electron-nucleus scattering cross section at $Q^{2} \sim 2-3(\mathrm{GeV} / \mathrm{c})^{2}$ (i.e. the region of energy loss $\nu<<Q^{2} / 2 m$, corresponding to $x>2, x=Q^{2} / 2 m \nu$ being the Bjorken scaling variable) has been shown to be dominated by processes in which the struck nucleon rescatters against the spectators within distances of the order of $1 \mathrm{fm}$ from the position of the electromagnetic vertex ${ }^{11,12}$. Over such a short lengthscale, CT is expectd to produce an appreciable suppression of the NN cross section even at moderate $Q^{2}$ and the inclusion of this effect brings theoretical calculations to good agreement with the data (see fig. 6). The upcoming inclusive data at $x>1$ from Jefferson Lab $^{14}$ will certainly shed light on this issue.

\section{Acknowledgments}

Most of the work discusssed in this paper has been carried out in collaboration with V.R. Pandharipande an S.C. Pieper.

\section{References}

1. T.G. O'Neill et al, Phys. Lett. B351(1995)87.

2. R. Ent et al, in "Electron Nucleus Scattering", Eds. O. Benhar and A. Fabrocini (ETS, Pisa, 1997)p. 378.

3. O. Benhar et al, Phys. Rev. Lett. 69, 881 (1992).

4. V.R. Pandharipande and S.C. Pieper, Phys. Rev. C 45, 791 (1992).

5. O. Benhar, in "Electron Nucleus Scattering", Eds. O. Benhar and A. Fabrocini (ETS, Pisa, 1997)p. 428.

6. S.C. Pieper, private communication.

7. H. de Vries et al. At. Data Nucl. Data Tables 36, 495 (1987).

8. S.C. Pieper, R.B. Wiringa and V.R. Pandharipande, Phys. Rev. Lett. 64, 364 (1990). 
9. H. Gao, private communication.

10. G. Garino et al, Phys. Rev. C 45, 780 (1992).

11. O. Benhar et al, Phys. Rev. C 44, 2328 (1991).

12. O. Benhar and S. Liuti, Phys. Lett. 389B, 649 (1996).

13. B.D. Day et al, Phys. Rev. C 40, 1011 (1989).

14. TJNAF experiment E89-008, spokespersons D.B. Day and B.W. Filippone.

\section{Figures}

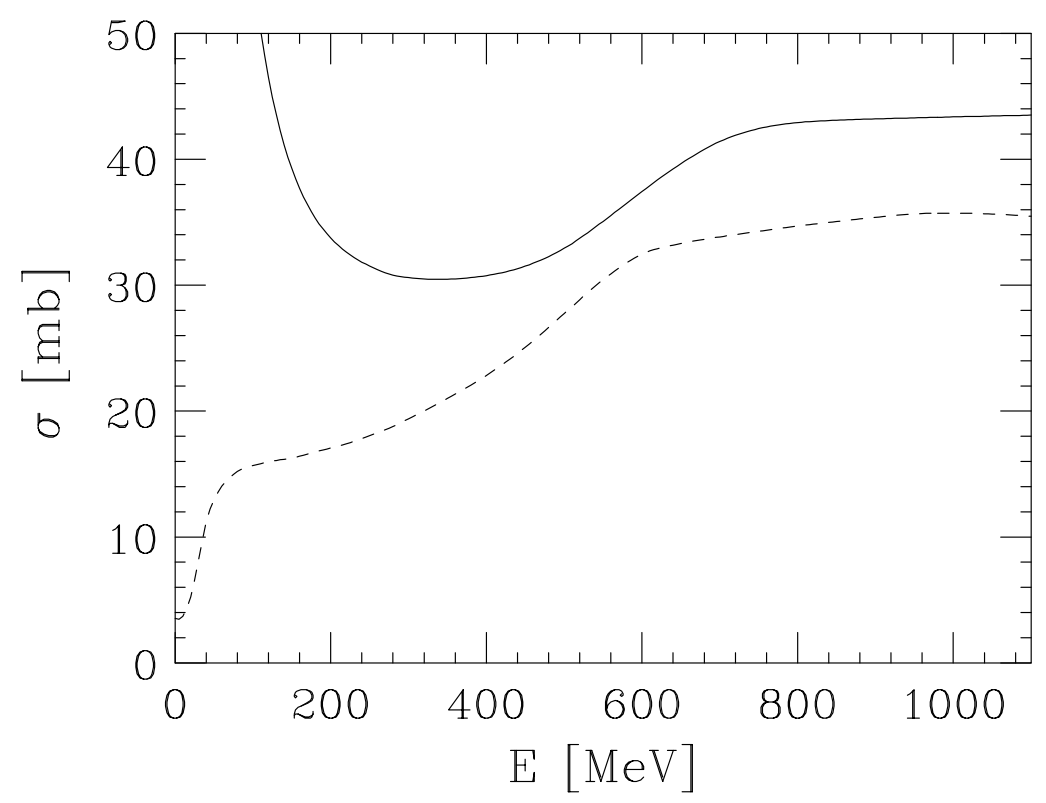

Figure 1: Free space (solid line) and in medium (dashed line) NN total cross section, as a function of the energy of the beam particle in the laboratory frame. 


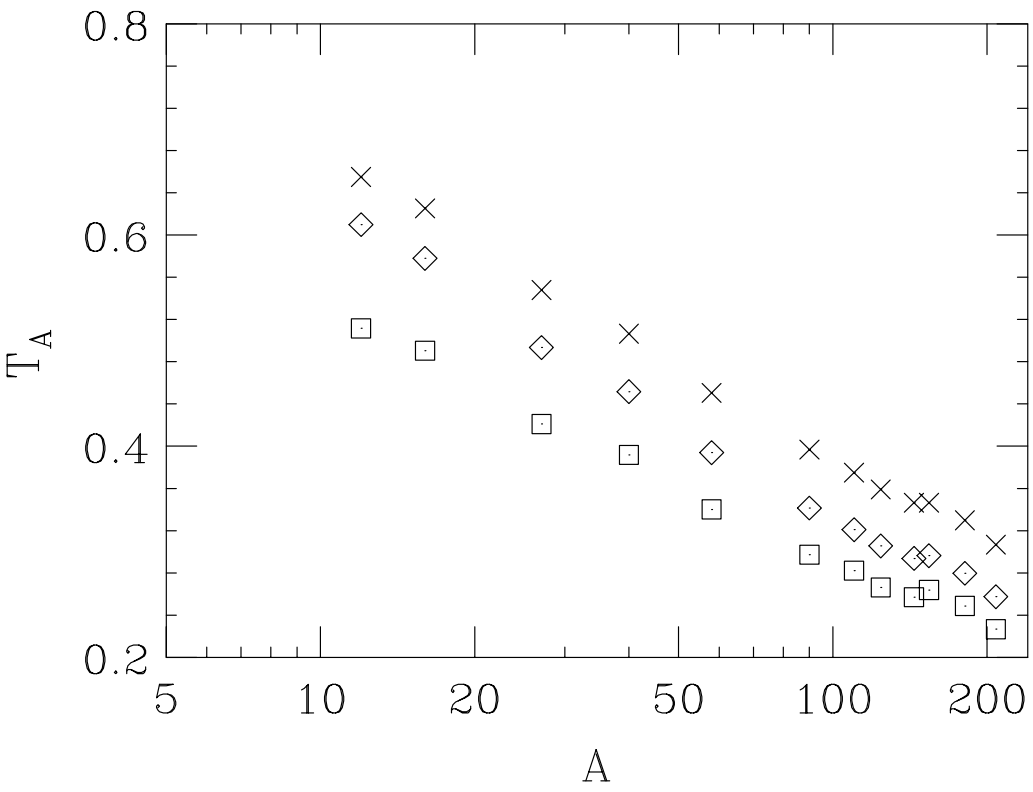

Figure 2: A-dependence of the nuclear transparency $T_{A}$ calculated including single- (crosses) and double-rescattering (diamonds) contributions. The result of the standard Glauber approximation (i.e. obtained neglecting the effect of NN correlations) are also shown (squares). 


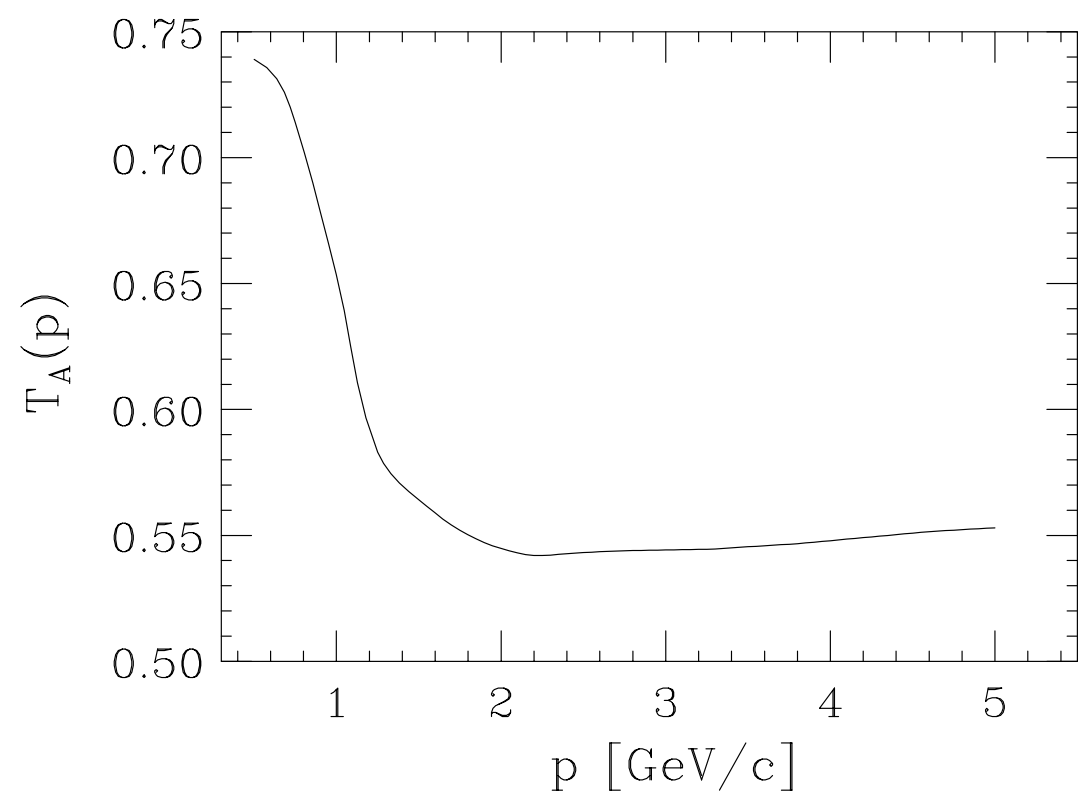

Figure 3: Momentum dependence of the ${ }^{16} \mathrm{O}$ transparency calculated from eq.(4), i.e. including all rescattering contributions, using the many-body wave function of ref. 8 . 


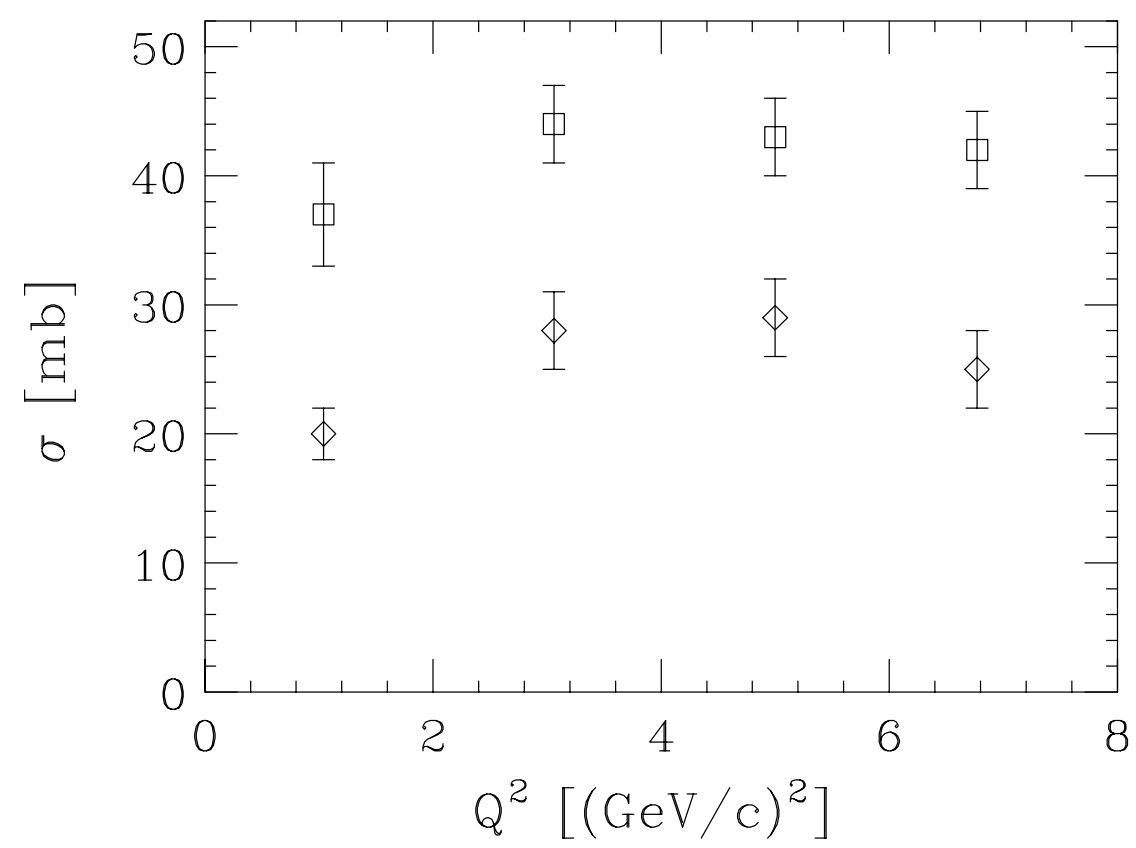

Figure 4: Comparison between the free-space NN total cross section (squares) and the effective cross section extracted from the analysis of the A-dependence of the data of ref. 1 (diamonds). 


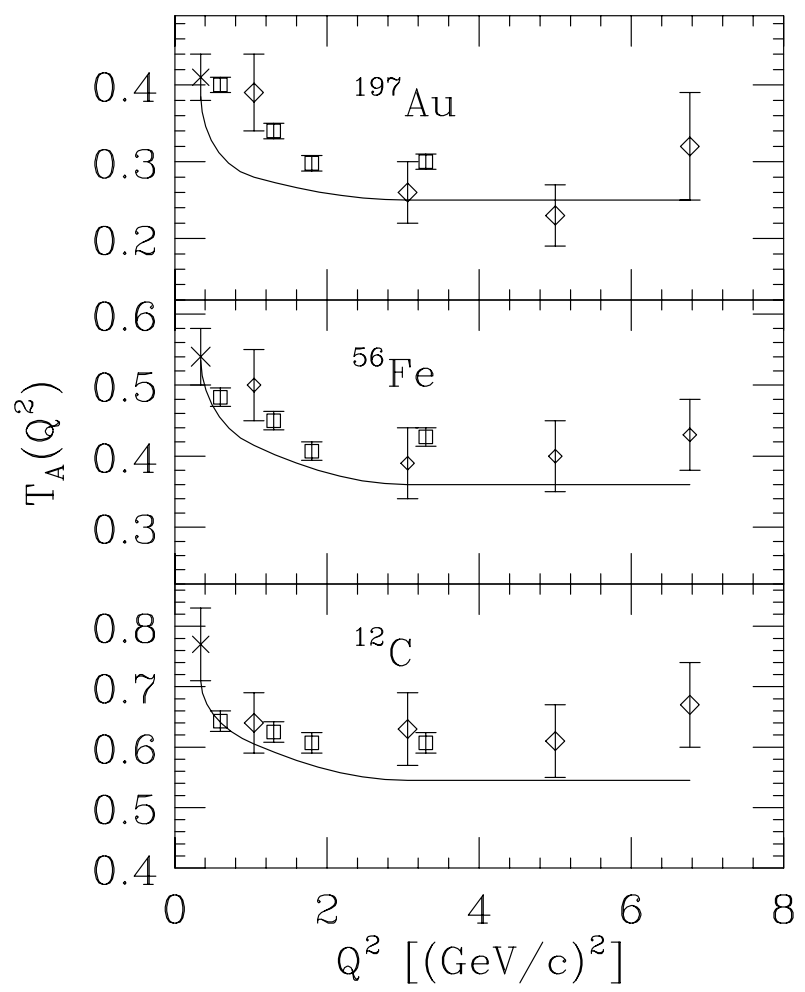

Figure 5: Comparison between the results of a many-body calculation of the nuclear transparency, carried out within the local density approximation and including the two- and three-rescattering contributions, and the available $\left(e, e^{\prime} p\right)$ data. Diamonds and squares correspond to the data of refs. 1 and 2 , respectively, whereas the crosses show the data of ref.

10. 


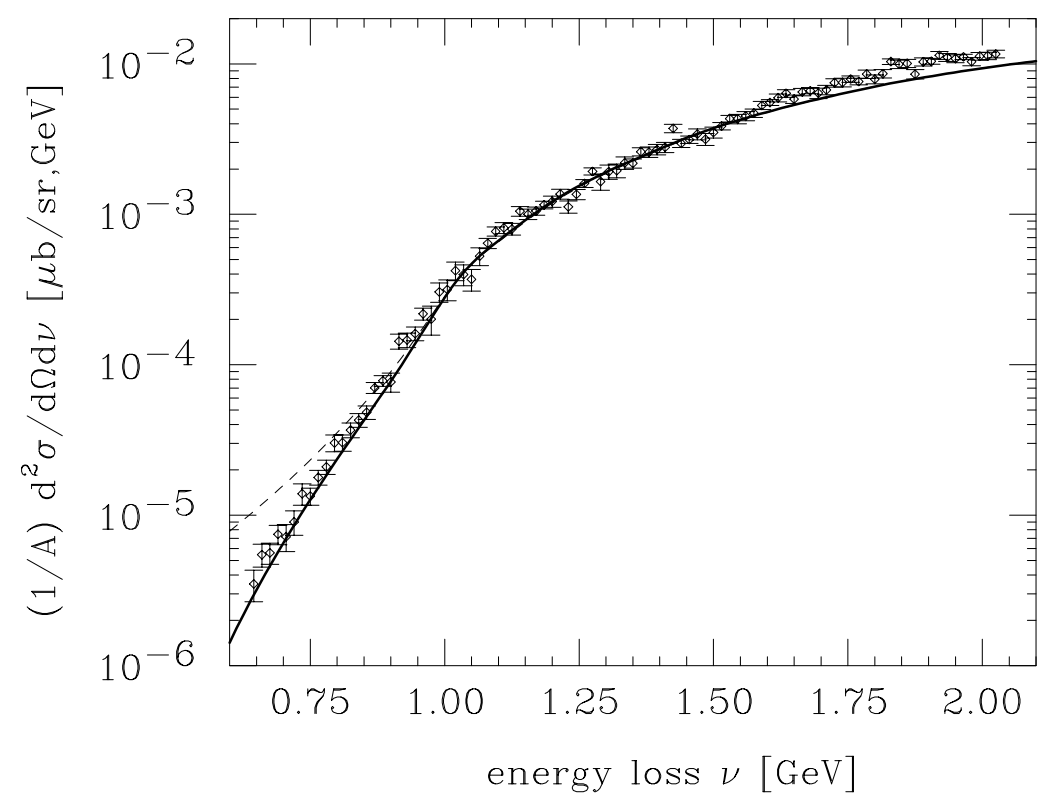

Figure 6: Extrapolated inclusive cross section off infinite nuclear matter (see ref. 13) at incident energy $E=3.595 \mathrm{GeV}$ and scattering angle $\theta=30^{\circ}$, plotted as a function of the electron energy loss $\nu$. The solid and dashed lines show the theoretical results of ref. 11, with and without inclusion of $\mathrm{CT}$, respectively. 